\title{
HETEROGENEOUS SOLID PROPELLANTS: FROM MICROSTRUCTURE TO MACROSCALE PROPERTIES
}

\section{S. Gallier}

SNPE Matériaux Energétiques

Vert-le-petit 91710, France

Composite solid propellants are inherently heterogeneous at microscopic level. Consequently, global mixture laws are often inappropriate because they cannot capture the underlying microstructural features. This paper intends to give an overview of some recent multiscale efforts in three different industrial issues related to solid propellants: combustion, electric breakdown, and aluminum agglomeration. For each physical process, direct simulations are performed at microscale level using sphere packs to model the propellant. Parametric computations help devising some global models that occur to be fairly well validated by experimental data. The benefit of such approach is that it can naturally account for microstructural features. In particular, it is shown that percolation process can often arise in composite propellants and significantly alter macroscopic properties.

\section{INTRODUCTION}

Heterogeneous solid propellants used in rocket industry are usually composed of crystalline particles, frequently ammonium perchlorate (AP), embedded in a polymeric binder (generally, HTPB: Hydroxyl-Terminated PolyButadiene). Aluminum particles are also often added in order to improve propulsive performances. A typical solid propellant, hence, consists of about $70 \% \mathrm{AP}$ in mass with wide size distributions, about $10 \%$ HTPB binder, and around $20 \%$ aluminum. Particle size may typically range from 1 to $100 \mu \mathrm{m}$.

This inherent heterogeneity at microscopic level may have a strong impact on macroscale properties and global mixture laws are often inappropriate because

This is an Open Access article distributed under the terms of the Creative Commons Attribution-Noncommercial License 3.0, which permits unrestricted use, distribution, and reproduction in any noncommercial medium, provided the original work is properly cited. 
they cannot capture such underlying microstructural features. This may be encountered in the field of detonation (e.g., hotspot formation during deflagrationto-detonation transition), combustion (e.g., aluminum agglomeration or fine AP/binder premixed flames), and mechanics (e.g., filler/binder adhesion), etc. This strongly motivates an improved knowledge of propellant microstructure as well as a multiscale strategy development. Yet, much multiscale approaches dedicated to solid propellants have focused on mechanics, through homogenization techniques, for instance. Thus, some aspects have received much less attention from this viewpoint of multiscale modeling. In particular, this is the case for combustion and aluminum agglomeration.

This study intends to give an overview of some recent multiscale simulation results on solid propellants. Some simulations are performed at microscale level and are liable to yield some macroscale mixture laws because they are more suitable for practical use. Three different industrial issues are dealt with in the present work. The first one considers some aspects of microstructural features at combustion of an ideal binary monomodal propellant. The second aspect is linked with electrostatic hazards because aluminized propellants are liable to undergo electrical breakdowns. Finally, the issue of aluminum agglomeration is tackled with a new model capable of predicting agglomeration based on the explicit description of propellant microstructure.

\section{MICROSTRUCTURE OF SOLID PROPELLANTS}

Solid propellants are modeled by a packing, which is basically a random arrangement of hard spheres in a cubic domain of fixed size $L$ (so-called RVE: Representative Volume Element). It allows accounting for multimodal propellants in a natural way as well as any particle size distributions. General and refined algorithms for packing can be found in the literature $[1,2]$ because it is a problem of importance for a wide variety of physical systems. In this study, the RSA algorithm from Torquato [3] will be primarily used. It is the most simple and CPU efficient algorithm, as it consists in placing randomly and sequentially nonoverlapping spheres: in other words, the coordinates of sphere center are randomized and the sphere is accepted if it does not overlap with previously accepted spheres. This algorithm is used for low packing fractions because it is unable to pack higher than $38 \%$ in volume [3]. When higher volume fractions are needed, a variant of the Jodrey-Tory algorithm [1] is also employed.

Periodic boundary conditions are generally used to minimize finite-system size effects although this is, however, known to affect high-order statistics, especially the pair-connectedness function [3]. In the present case, an embedded cell approach is used when a packing is performed in a large RVE (with wall boundary conditions), inside which a smaller RVE is sampled. This latter RVE is consid- 
ered for ensuing computations. Its size must be at least a factor of ten larger than particle diameter $D$ to ensure statistical representativity.

The relevance of such approach to model a real composite solid propellant was recently investigated by the present author and a thorough comparison was performed between packings and experimental data obtained via X-Ray microtomography [4]. For the AP/HTPB propellant considered, statistical parameters — such as nearest-neighbor distance distribution or pair-correlation function - were in fairly correct agreement with experiments, which suggests

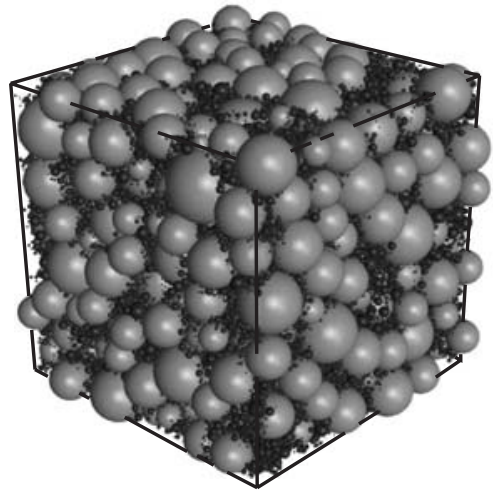

Figure 1 Example of a propellant pack that such sphere packs should be representative of actual propellant structures. An example of such a computed microstructure is presented in Fig. 1. Coarse AP particles (mean size $200 \mu \mathrm{m}$ ) are here displayed in gray whereas aluminum particles are displayed in black. The RVE is $L=2 \mathrm{~mm}$ large and includes roughly 100.000 particles (mostly, aluminum).

\section{MULTISCALE MODELING IN PROPELLANT COMBUSTION}

\subsection{Introduction}

A reliable prediction of solid propellant combustion is of great importance for industrial applications because propellants for propulsion applications must meet strict requirements as far as ballistic properties are concerned (e.g., burning rate or pressure exponent). However, the complexity of solid propellant combustion has hindered the development of reliable predictive models, which explains the persistent popularity of zero-dimensional (0D) global models. Nevertheless, three-dimensional (3D) microscale simulations have recently emerged and given propellant combustion a new lease of life $[5,6]$. They give brand new insights in combustion physics and improve prediction capabilities, but at the cost of huge CPU resources and limited types of propellant handled.

The recent work [7] tried to draw a bridge between simple 0D models and $3 \mathrm{D}$ detailed simulations. The purpose was not to substitute for such detailed modeling but rather to focus on how the combustion front propagation (and, hence, the burning rate) can be modified by the microstructure. 


\subsection{Front Propagation Model}

The problem may be recast in the following form: consider an ideal binary composite propellant with oxidizer particles, loaded at volume fraction $\xi$, and binder (at fraction $1-\xi$ ). Let $r_{\mathrm{ox}}$ (respectively, $r_{b}$ ) be the burning rate of neat oxidizer (respectively, binder). The question is then to determine the burning rate $r_{p}$ of this mixture, assuming that burning rates $r_{\text {ox }}$ and $r_{b}$ are already known (for instance, through a 0D simple monopropellant model).

Various approaches have been developed in the literature to propose such a mixture law (space-averaging techniques, geometrical model, relay-race model, etc.) and most of them are based on statistical considerations of the propellant structure. The variety of models developed to cope with this problem suggests that it might not be so simple. The present approach consists in modeling the composite propellant as a random arrangement of spherical particles that will be "numerically burnt" by some surface evolution computation. This supposes that both burning rates for oxidizer and binder are prescribed. Imposing burning rates for oxidizer and binder suggests they burn on their own, which might be of limited practical interest in some cases (for instance, when diffusion flames are present). However, this is a deliberate choice since the present study does not intend to develop a complex predictive model but rather aims at setting a mixture law which incorporates, though in a simple way, geometrical information on propellant microstructure.

In order to simulate propellant regression, it is chosen to use a level-set method. The main advantage is that complex topological surface changes are easily handled because the front is implicitly captured by a fixed Eulerian grid. Let $\psi(\mathbf{X}, t)$ be a level-set function such that $\psi(\mathbf{X}, t)>0$ in the burnt region and $\psi(\mathbf{X}, t)<0$ in the unburnt region. The combustion front is naturally associated to the hypersurface defined by the locus $\psi(\mathbf{X}, t)=0$. As the front propagates along its normal direction, with local rate $r(\mathbf{X})$, then the level-set function follows a Hamilton-Jacobi "type" equation:

$$
\frac{\partial \psi}{\partial t}+r(\vec{X})|\vec{\nabla} \psi|=0
$$

This equation is discretized on a 3D Cartesian grid using a fifth-order accurate WENO (weighted essentially nonoscillatory) scheme. Some details on the numerical procedure can be found in [7].

For a given packing (generated using the algorithms detailed in the previous section), it is postulated that spheres represent oxidizer particles and are prescribed to burn with rate $r_{\mathrm{ox}}$. The remaining space is assumed to model the binder matrix, regressing at rate $r_{b}$. It is supposed that burning starts from the top of the RVE. Surface regression Eq. (1) is then evolved in time until propellant is burnt out, from which the burning time and average burning rate are estimated. 


\subsection{Front Propagation Results}

Monomodal packings with different particle volume fraction $\xi$ (typically, from 0.05 to 0.6 ) are generated and surface evolution and burning rates are computed accordingly, with various oxidizer-to-binder rate ratio $Z=r_{\mathrm{ox}} / r_{b}$. The "mixture law" $R=R(\xi, Z)$ is then obtained numerically, where $R$ is the nondimensional propellant burning rate $R=\left(r_{p}-r_{b}\right) /\left(r_{\mathrm{ox}}-r_{b}\right)$. In the present computations, $L=1 \mathrm{~mm}, D=100 \mu \mathrm{m}$, and a mesh with $150^{3}$ grid points was used. The detailed computational procedure can be found in [7].

The present computations suggest the existence of three different behaviors, depending on the values of the burning rate ratio $Z$. When the oxidizer and binder burn at the same rate $(Z \sim 1)$, the propellant burning rate $R$ depends linearly on the oxidizer fraction: $R=\xi$. This involves the dimensional burning rate law $1 / r_{p}=\xi / r_{\mathrm{ox}}+(1-\xi) / r_{b}$, which is actually often used (sometimes referred to as the "slab-layer model").

However, when the burning rate ratio $Z$ is increased, the burning rate $R$ departs from linearity by taking a more concave shape. This means that the theoretical formalism of the previous slab-layer model is only valid if the combustion front is absolutely plane (limit case $Z=1$ ), which is of little practical interest. Figure $2 a$ shows the burning rate $R$ for the typical values $1.25<Z<3$. A striking feature is that all the rates collapse into a single curve for this range of $Z$ values. This means that a kind of universal law stands and is explicitly independent on the burning rate ratio $Z$. This law has been checked to be in good agreement with some experimental data [7].

Yet, the burning rate gradually tends to the S-shaped curve with increasing $Z$, as seen in Fig. $2 b$. The limit case $Z=\infty$ is also investigated by setting $r_{b}=0$. In this case, Fig. $2 b$ clearly shows that regression is only possible above a threshold particle volume fraction. The actual reason for this change is that the oxidizer

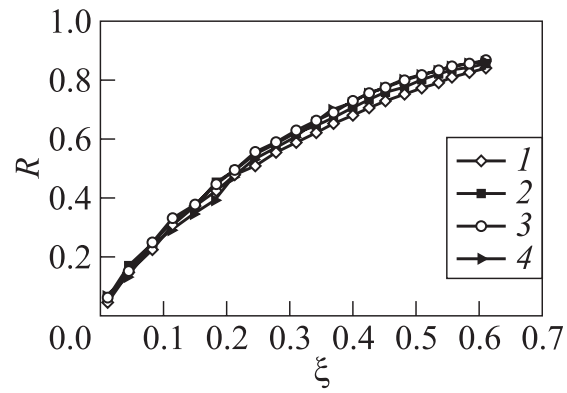

(a)

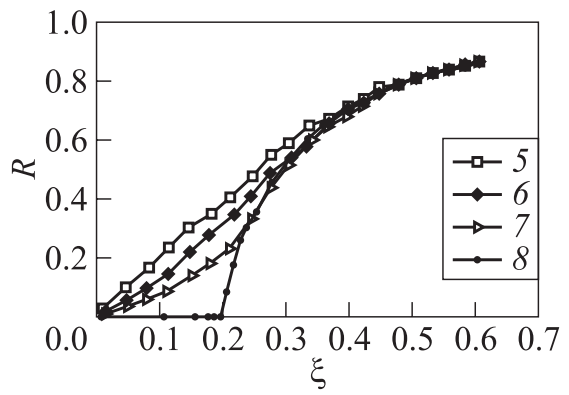

(b)

Figure 2 Computed burning rate vs. volume fraction: $1-Z=1.25 ; 2-1.5 ; 3-$ $2 ; 4-3 ; 5-5 ; 6-10 ; 7-20$; and $8-Z=\infty$ 


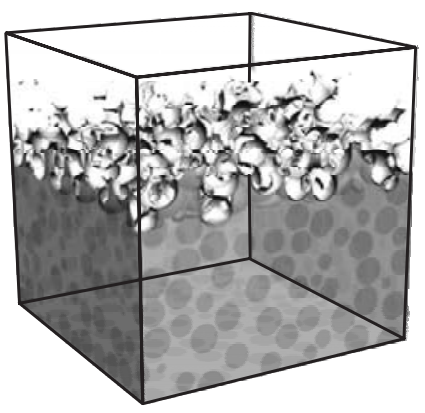

Figure 3 Snapshot of combustion front $(Z=10$ and $\xi=0.5)$

burns so fast compared to binder that combustion takes place mostly through adjacent particles.

Figure 3 displays an instantaneous view of the combustion front for this regime $(Z=$ 10 and $\xi=0.5)$. A complex combustion surface can be seen with crater-like holes due to burnt out particles. This is undoubtedly a different combustion mode where the oxidizer has an enhanced effect and binder has virtually no role. The results from Fig. $2 b$ for $Z=\infty$ are indeed typically expected from percolation theory and combustion can only progress when above a certain percolation threshold $\xi_{\mathrm{cr}}$. Beyond this critical value, combustion takes place only through a connecting path of particles, as the binder is nonburning for this case. This percolation regime is obviously closely connected to the spatial arrangement of particles at the microscale level. The computed percolation threshold is found to be rather close to the universal critical threshold $\xi_{\text {cr }} \sim 0.15$ for 3D structures (Scher-Zallen invariant [8]). The present results are also in good agreement with some Russian data on the combustion of binary mixtures, for which a rapid upturn in the burning rate is noted by $\xi_{\mathrm{cr}} \sim 0.16[9]$.

It is worth here to again insist on the fact that the previous model is not a predictive full gasdynamical model like in $[5,6]$ but it allows isolating the (important) role the microstructure can have on the combustion front propagation. It is, however, believed that this microstructure-based approach is suitable for systems devoid of diffusion flames, where binder and oxidizer burn on their own without significant mutual influence.

\section{MULTISCALE MODELING IN DIELECTRIC BREAKDOWN}

\subsection{Introduction}

From an electrical point of view, an aluminized solid propellant is a metal-loaded dielectrics since it can be seen as an insulating matrix (binder together with AP crystalline particles) with some metallic conducting inclusions of aluminum. Thereby, it is liable to experience a dielectric breakdown when submitted to an intense electric field, just like a capacitor does. In this case, this may induce the ignition, possibly followed by the deflagration of the solid propellant itself. 
This large sensitivity of solid propellants to electrostatic discharge is a major safety concern and the prediction of this sensitivity is crucial for the production, handling, and operation of solid rocket motors.

It has been observed [10] that the breakdown field is considerably lowered by the presence of aluminum particles and is a strong function of aluminum size and content, which suggests a predominant role of the microstructure. The effects of microscale structure have been theoretically confirmed by Beale and Duxbury [11] who considered some lattice models of conductor/insulating bonds. This approach showed that the breakdown is controlled by a percolation regime and that the breakdown electric field $E_{\text {break }}$ goes to 0 when approaching a critical volume fraction $\xi_{\mathrm{cr}}$ for conductors. Later on, preliminary electrostatic computations have been carried out at the microscale level by Gyure and Beale [12]. Due to the limited CPU resources at that time, the computations were mainly two-dimensional (2D) (disks) and with a limited number of conductors (a few tens). However, a major result was that the breakdown field $E_{\text {break }}$ is directly connected to the mean nearest-neighbor distance $d$ between particles through the following simple relation (noting $E_{\text {binder }}$ the binder dielectric strength and $D$ the particle diameter):

$$
\frac{E_{\text {break }}}{E_{\text {binder }}}=\frac{d}{d+D} .
$$

The present study intends to take advantage of modern numerical techniques and computing performances to revisit the problem of electrical breakdown in solid propellants. One feature of this work is to systematically consider 3D microstructures, with a number of particles one or two orders of magnitude larger compared to Gyure's works, and to extend this analysis to real solid propellants.

\subsection{Electrostatic Model}

Just like the previous combustion problem, some monomodal packs are generated with the present packing algorithm. Particles are assumed to represent aluminum (electrical conductivity $\sigma_{\mathrm{Al}}$ ) and the remaining space is the matrix that lumps together the binder and the crystalline particles (electrical conductivity $\sigma_{\text {Matrix }}$ ). The steady electrostatic problem, in absence of distributed sources, is ruled by the following Maxwell equation (where $\varphi$ is the electrostatic potential):

$$
\nabla \cdot(\sigma \nabla \varphi)=0 .
$$

This variable-coefficient Laplace equation is directly solved in the $3 \mathrm{D}$ generated microstructure, with the imposed potential on top and bottom surfaces that corresponds to a prescribed external field $E_{0}$. A finite-difference technique is employed with a standard centered second-order stencil and the resulting linear system is inverted using a BiCGstab solver with multigrid preconditioning. 


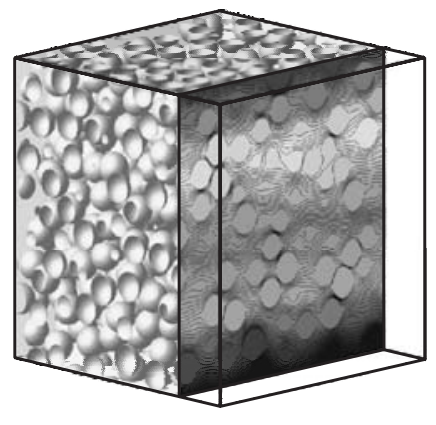

Figure 4 Equipotentials in a monodisperse pack
Some details on the numerical procedure as well as validations performed with this approach can be found in [13]. Figure 4 displays an example of the computed equipotentials $\varphi$ inside a monodisperse pack loaded at $30 \%$ in volume $\left(\sigma_{\mathrm{Al}} / \sigma_{\text {Matrix }}=10^{6}\right)$. Note that between very close spheres, isolines are squeezed together, involving large local electrical fields $E=-\nabla \varphi$.

Breakdown dynamics in a solid propellant has been experimentally conjectured to arise from local breakdowns progressing through adjacent conducting particles [10]. Indeed, the electrostatic field among two close particles can be large enough to promote the breakdown of the small amount of dielectrics in-between.

The resulting physical mechanism is presumably a vaporization of a portion of the metallic particles followed by resolidification as a single conductor. Thus, those two conductors will share charge and attain the same electrical potential. Consequently, new local breakdowns can happen and, at the end, result in a likely top-to-bottom conducting path, followed by a disruptive discharge through the material. Following this mechanism, all pairs $(i, j)$ of particles are considered and the electric field $E_{i j}$ in-between can be easily computed since Eq. (3) has been solved. If this local field exceeds the binder breakdown strength $E_{\text {binder }}$, then a local breakdown is supposed to take place. Following the physics assumed, it is surmised that these two particles will attain the same potential. This is numerically done by connecting both particles with a thin high-conductivity bond. Then, the computation of the whole field (Eq. (3)) is resumed, so that these two particles will now attain the same potential. As a result, a new local breakdown is prone to happen and this sequence of breakdowns/field updates is iterated until the breakdown path either stops or spans the domain. In the latter case, the sample is supposed to experience a global breakdown. In the former case, the imposed external field $E_{0}$ is increased until a breakdown path effectively forms a top-to-bottom connection. If so, the field $E_{0}$ is supposed to be the propellant breakdown strength $E_{\text {break }}$.

\subsection{Results}

Monomodal packs with different volume fraction $\xi$ are generated and the electrostatic problem is solved for the case of superconducting inclusions $\left(\sigma_{\mathrm{Al}} / \sigma_{\mathrm{Matrix}}\right.$ $\left.=10^{6}\right)$. Binder breakdown strength is set to $E_{\text {binder }}=20 \mathrm{MV} / \mathrm{m}$ and computations are performed on a $N=129^{3}$ grid point mesh. In order to check the 
relevance of Gyure relationship (Eq. (2)), the results are displayed in terms of the mean nearest-neighbor distance $d$. Figure 5 shows the present simulation results together with some experimental data from Duxbury et al. [14] obtained on steel balls embedded in wax. In present case, the RVE size is chosen to $L=1 \mathrm{~mm}$ with $D=100 \mu \mathrm{m}$ particles in order to comply with experiments [14]. Present computations are in very good accordance with the experiments but also with the simple law of Eq. (2) (dashed line in Fig. 5). It is interesting to notice that this law, postulated by Gyure and Beale [12] using 2D computations on as few as 25 disks, happens to remain valid when considering several hundreds of spheres. The importance of parameter $d /(d+D)$ suggests that the breakdown does progress from one particle to the closest particle because the largest field is expected there. It also means that breakdown in propellants is closely linked to the microstructure as it is governed by second-order space statistics parameters.

Surprisingly, computations for $\xi>0.2$ could not be performed because a conducting spanning path was detected at the first stage of the computation; thereby preventing any breakdown to take place. It happens that this can be ascribed to conducting particles forming, by themselves, a percolation cluster from top to bottom. This is reminiscent of the combustion problem addressed in the previous section. It means that the percolation process and cluster formation are a major microstructural feature that can play a significant role when studying composite propellants. This percolation threshold is also confirmed by many experimental works that have clearly showed that the switching between conducting and insulating behavior, for spherical conducting inclusions randomly dispersed in an insulating medium, is between 0.15 and 0.2 in volume fraction.

A direct electrostatic simulation on a real propellant would require colossal CPU resources due to the large size mismatch between the coarsest AP and the finest aluminum particles (see, e.g., Fig. 1). Rather, it is preferred to apply

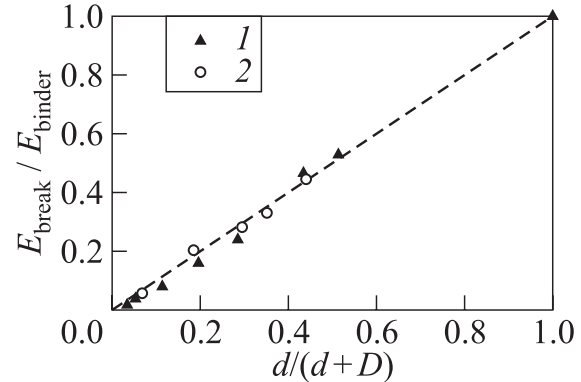

Figure 5 Breakdown strength vs. $d /(d+D): 1$ - computations; and 2 experiments

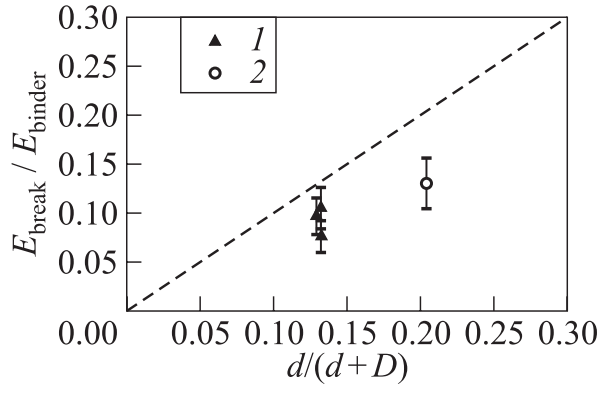

Figure 6 Experimental breakdown strengths: $1-5$-micron $\mathrm{Al}$; and $2-15$ micron $\mathrm{Al}$ 
the validated law from Eq. (2). Four industrial propellants have been considered and packed (Fig. 1 shows an example of one of the tested propellants). These four propellants differ from AP size as well as aluminum size (diameter 5 and $15 \mu \mathrm{m}$ ). Aluminum volume fraction is about 0.1 . Breakdown strengths have been experimentally measured on cylindrical samples of propellants and results are compared with mixture law prediction (dashed line) in Fig. 6. The general trend anticipated by Eq. (2) seems to be respected as the propellant with the highest $d /(d+D)$ (15-micron aluminum) shows the largest strength. However, the correlation is much less accurate compared to the previous idealized monodisperse mixture (see Fig. 5). It can be concluded that Eq. (2) is reasonably accurate to get an order of magnitude of the breakdown strength for a given propellant. Yet, it seems that the complexity of real propellant structures cannot perfectly comply with such a simple formulation. Possible reasons to explain the noted discrepancy can be attributed to particle shape (not exactly spherical) or finite-size effects in percolation process.

\section{MULTISCALE MODELING IN AGGLOMERATION}

\subsection{Introduction}

During combustion of aluminized propellants, agglomeration of aluminum at the burning surface is likely to happen. Aluminum particles may sinter and/or coalesce as the heat wave passes by and this may lead to agglomerates with a size much larger than the initial virgin aluminum introduced in the propellant. Large agglomerates will have a substantial distributed combustion that may significantly alter expected performances (two-phase losses, slag accumulation, instabilities, etc.).

Agglomeration has been the focus of many studies so far that have stressed the complexity of this process and hindered the development of reliable predictive models. On the one hand, tentative models describing the physics of agglomeration have been proposed by Russian scientists $[15,16]$ but they come up against the difficulty of modeling surface adhesion schemes, liquid particle movement on surface, etc. On the other hand, a very simple and attractive approach is the pocket model $[17,18]$. The key point is the notion of pockets, formed by the coarsest oxidizer particles, with aluminum particles encapsulated inside. According to such models, all the aluminum particles located inside a pocket coalesce and are united into a single agglomerate. More recently, Jackson et al. [19] proposed a new class of agglomeration models based on packings, giving full information on the locations of aluminum particles. Basically, two aluminum particles are supposed to coalesce if a certain (experimentally fitted) geometrical criterion is fulfilled. 
The present approach is to propose a revisited pocket model, in which the concept of pocket is retained, but where relevant geometrical information would be extracted from a packing. In one sense, it is a hybrid model between pocket models [18] and packing-based models [19].

\subsection{Hybrid Pocket/Packing Model}

The idea is to consider, from a statistical point of view, the spatial arrangement of the aluminum particles in the packing (it is assumed that such structure is isotropic and homogeneous). To do so, the $K$-Ripley function [20] will be used, which is the average number of neighboring particle, within distance $r$ from a typical particle, divided by the particle density $\rho$. For a $3 \mathrm{D}$ random arrangement, it could be expected that $K(r)=4 / 3 \pi r^{3}$ and values of $K(r)$ greater (respectively, lower) than $4 / 3 \pi r^{3}$ will indicate clustered (respectively, regular) structures. Hence, computing function $K(r)$ will help detecting the spatial concentration or dispersion of aluminum particles and, hence, pockets. It is common to use the following normalized version of $K$-function (so-called $L$-Besag function):

$$
L(r)=\sqrt[3]{\frac{3 K}{4 \pi}}-r .
$$

This function $L$ is helpful to reveal the spatial structure because it peaks at an average cluster radius: this is nothing else than the mean size of pockets. If one notes as $r_{\text {ag }}$ the value maximizing the $L$ function, then the expected number of aluminum particles $N\left(r<r_{\text {ag }}\right)$ inside this aggregation region (or pocket) is by definition $N\left(r<r_{\mathrm{ag}}\right)=\rho K\left(r_{\mathrm{ag}}\right)$. It can be easily shown that the total volume of aluminum in this aggregation zone $V\left(r<r_{\mathrm{ag}}\right)$ is given by: $V\left(r<r_{\mathrm{ag}}\right)=\xi K\left(r_{\mathrm{ag}}\right)$ with $\xi$ the aluminum volume fraction. The basic assumption of the pocket model is that all the previous aluminum volume contained inside one pocket is united into a single particle. This forms a so-called "potential" diameter $D_{\text {pot }}$ of the equivalent agglomerate and reads:

$$
D_{\text {pot }}=2 \xi^{1 / 3}\left(L\left(r_{\mathrm{ag}}\right)+r_{\mathrm{ag}}\right) .
$$

More details on setting Eq. (4) can be found in [21].

\subsection{Agglomeration Results}

This model has been applied to five different $\mathrm{AP} / \mathrm{Al} / \mathrm{HTPB}$ propellants. The packing is first generated for these five propellants and the potential agglomeration diameter is obtained via Eq. (4). Investigated propellants have all $68 \%$ AP 


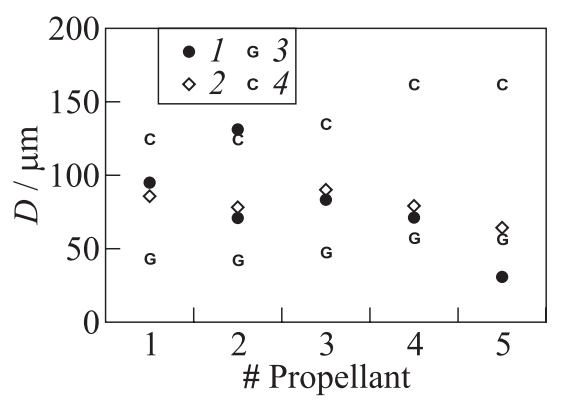

Figure 7 Predicted agglomeration diameters: 1 - experimental; 2 - present computation; $3-[17]$; and $4-[18]$

in mass and $18 \% \mathrm{Al}$. Noting $\mathrm{C}$ for coarse $(200 \mu \mathrm{m}) \mathrm{AP}, \mathrm{M}$ for medium $(100 \mu \mathrm{m})$, $\mathrm{F}$ for fine $(5 \mu \mathrm{m}), \mathrm{Al}_{\mathrm{C}}$ for coarse aluminum $(40 \mu \mathrm{m})$, and $\mathrm{Al}_{\mathrm{F}}$ for fine $(5 \mu \mathrm{m})$, the formulations are:

$$
\begin{gathered}
\# 1=\left(58 \% \mathrm{C} / 10 \% \mathrm{~F} / 18 \% \mathrm{Al}_{\mathrm{C}}\right) ; \quad \# 2=\left(58 \% \mathrm{C} / 10 \% \mathrm{~F} / 18 \% \mathrm{Al}_{\mathrm{F}}\right) ; \\
\# 3=\left(48 \% \mathrm{C} / 20 \% \mathrm{~F} / 18 \% \mathrm{Al}_{\mathrm{C}}\right) ; \quad \# 4=\left(28 \% \mathrm{C} / 25 \% \mathrm{M} / 15 \% \mathrm{~F} / 18 \% \mathrm{Al}_{\mathrm{C}}\right) ; \\
\# 5=\left(28 \% \mathrm{C} / 25 \% \mathrm{M} / 15 \% \mathrm{~F} / 18 \% \mathrm{Al}_{\mathrm{F}}\right) .
\end{gathered}
$$

More details can be found in [21].

Figure 7 presents the present results against experimental data. Also displayed are the predictions from the Grigoriev [17] and Cohen [18] pocket models. It seems that the proposed model is capable of correctly predicting the measured agglomerate diameters, at least for the five propellants tested (for propellant \#2, the two experimental data correspond to two peaks noted in the diameter distribution). In particular, the correct trend is predicted, which is not the case for the other pocket models. The present model offers improved predictions, compared to classical pocket models, because it accounts for the real propellant microstructure. For instance, pocket models predict a similar agglomeration for propellants \#4 and \#5 whereas the present microstructure-based model anticipates a reduction in agglomeration due to an improved dispersion of fine aluminum particles.

A similar comparison has also been applied to the agglomeration ratio [21]. Here again, it seems that a microstructure-based model is more representative that a classical pocket approach. The downside is, however, an increase in CPU time due to packing generation (for fine aluminum packs, several million particles are needed for statistical relevance).

It can be argued that keeping a pocket assumption might limit the applicability of the model. This is true to some extent because, by construction, this model cannot predict some experimental facts (e.g., the effects of burn rate or binder 
nature on agglomeration) and is, moreover, only capable of estimating a single agglomerate size rather than size distribution. Obviously, extra work is needed. Despite these limitations, the model stresses here again a major role of the propellant microstructure and is furthermore capable of giving correct "blind-test" predictions without a posteriori calibration (because it is parameter-free), which may not be the case for more complex models.

\section{CONCLUDING REMARKS}

This work presented an overview of some recent multiscale modeling in different issues related to composite solid propellants, namely, combustion, electrical breakdown and aluminum agglomeration.

The problems of combustion and electrical breakdown are treated in a very similar way. Basically, monodisperse sphere packs are used to model the propellant microstructure and direct computations are performed in the resulting $3 \mathrm{D}$ RVE. Parametric computations, in particular, on particle volume fraction, have allowed setting global mixture laws for the desired macroscale properties (burning rate or dielectric strength) while accounting for the inherent microstructural features. The obtained mixture laws seem to be globally well validated by experimental data. An interesting feature is that both problems seem to be controlled by percolation in some cases. The critical threshold is about 0.20 in volume, quite close to current know-how, above which a dramatic change in macroscale properties is observed due to the formation of a connected particle path (involving burning rate enhancement or disappearing of breakdowns).

For aluminum agglomeration, a new approach has been proposed that combines the idea of pocket models and geometrical information from packings. Some space statistics then provides an average pocket volume and agglomerate size. The obtained model is parameter-free and outperforms simple pocket models since it relaxes any assumption on the propellant structure.

In all cases, it is numerically demonstrated that a microstructure-based approach is liable to bring new insights into the complex physics of composite propellants and is capable of providing simple but reliable macroscale laws that are more convenient to use in an industrial frame.

\section{REFERENCES}

1. Jodrey, W. S., and E. M. Tory. 1985. Computer simulation of close random packing of equal spheres. Phys. Rev. A 32:2347-51.

2. Lubachevsky, B. D., and F. H. Stillinger. 1990. Geometric properties of random disk packings. J. Stat. Phys. 60(5):561-83. 
3. Torquato, S. 2001. Random heterogeneous materials. Springer.

4. Gallier, S., and F. Hiernard. 2008. Microstructure of composite propellants using simulated packings and X-ray tomography. J. Propul. Power 24(1):154-57.

5. Jackson, T. L., and J. Buckmaster. 2002. Heterogeneous propellant combustion. AIAA J. 40(6):1122-30.

6. Massa, L., and T.L. Jackson. 2008. Multidimensional numerical simulation of ammonium-perchlorate-based propellant combustion with fine/ultrafine aluminum. J. Propul. Power 24(2):161-74.

7. Gallier, S., and J. F. Guéry. 2009. Regression fronts in random sphere packs: Application to composite solid propellant burning rate. Proc. Combust. Inst. 32(2):211522 .

8. Scher, H., and R. Zallen. 1970. Critical density in percolation processes. J. Chem. Phys. 53:3759-61.

9. Frolov, Yu., and A. Pivkina. 2007. High energy condensed systems. EuroPyro'07. Beaune, France.

10. Kent, R., and R. Rat. 1985. Static electricity phenomena in the manufacture and handling of solid propellants. J. Electrostat. 17:299-312.

11. Beale, P. D., and P. M. Duxbury. 1988. Dielectric breakdown in metal-loaded dielectrics. Phys. Rev. B 37:2785-91.

12. Gyure, M.F., and P.D. Beale. 1989. Dielectric breakdown of a random array of conducting cylinders. Phys. Rev. B 40(14):9533-40.

13. Gallier, S. 2010. Numerical modeling of dielectric breakdown in solid propellant microstructures. Int. J. Multiscale Comp. Eng. 5:523-33.

14. Duxbury, P. M., P. D. Beale, H. Bak, and P. A. Schroeder. 1990. Capacitance and dielectric breakdown of metal-loaded dielectrics. J. Phys. D 23:1546-53.

15. Babuk, V., I. Dolotkazin, and V. Sviridov 2003. Simulation of agglomerate dispersion in combustion of aluminized solid propellants. Combust. Explosion Shock Waves 39(2):195-203.

16. Rashkovskii, S. 2005. Statistical simulation of aluminum agglomeration during combustion of heterogeneous condensed mixtures. Combust. Explosion Shock Waves 41(2):174-84.

17. Grigoriev, V., K. Kutsenogii, and V. Zarko. 1981. Model of aluminum agglomeration during the combustion of a composite propellant. Combust. Explosion Shock Waves 17(4):356-62.

18. Cohen, N. 1983. A pocket model for agglomeration in composite propellants. AIAA J. 21(5):720-25.

19. Jackson, T., F. Najjar, and J. Buckmaster. 2005. A new class of agglomeration models for aluminum composite propellants based on random packs. J. Propul. Power 21(5):925-36.

20. Stoyan, D., W. Kendall, and J. Mecke. 1995. Stochastic geometry and its applications. 2nd ed. Wiley.

21. Gallier, S. 2009. A stochastic pocket model for aluminum agglomeration in solid propellants. Prop. Expl. Pyro. 34(2):97-105. 